\title{
Cyclodextrin-PEG conjugate-wrapped magnetic ferrite nanoparticles for enhanced drug loading and release
}

\author{
Israel V. M. V. Enoch ${ }^{1,2} \cdot$ Sivaraj Ramasamy $^{1} \cdot$ Shanid Mohiyuddin $^{3} \cdot$ Packirisamy Gopinath $^{3} \cdot$ R. Manoharan ${ }^{2}$
}

Received: 3 August 2017 / Accepted: 27 April 2018 / Published online: 25 May 2018

(c) Springer-Verlag GmbH Germany, part of Springer Nature 2018

\begin{abstract}
Magnetic nanoparticles are envisaged to overcome the impediments in the methods of targeted drug delivery and hence cure cancer effectively. We report herein, manganese ferrite nanoparticles, coated with $\beta$-cyclodextrin-modified polyethylene glycol as a carrier for the drug, camptothecin. The particles are of the size of $\sim 100 \mathrm{~nm}$ and they show superparamagnetic behaviour. The saturation magnetization does not get diminished on polymer coverage of the nanoparticles. The $\beta$-cyclodextrin-polyethylene glycol conjugates are characterized using NMR and mass spectrometric techniques. By coating the magnetic nanoparticles with the cyclodextrin-tethered polymer, the drug-loading capacity is enhanced and the observed release of the drug is slow and sustained. The cell viability of HEK293 and HCT15 cells is evaluated and the cytotoxicity is enhanced when the drug is loaded in the polymer-coated magnetic nanoparticles. The noncovalent-binding based and enhanced drug loading on the nanoparticles and the sustained release make the nanocarrier a promising agent for carrying the payload to the target.
\end{abstract}

Keywords Cyclodextrin $\cdot$ PEG $\cdot$ Camptothecin $\cdot$ Manganese ferrite $\cdot$ Drug release

\section{Introduction}

Magnetic nanoparticle (MNP)-polymer hybrids are versatile platforms for multiple functions, such as treatment, diagnosis, and monitoring of diseases, including cancer (Lee

Israel V. M. V. Enoch and Sivaraj Ramasamy have equally contributed.

Electronic supplementary material The online version of this article (https://doi.org/10.1007/s13204-018-0798-5) contains supplementary material, which is available to authorized users.

Israel V. M. V. Enoch

drisraelenoch@gmail.com

Packirisamy Gopinath

nanogopi@gmail.com

$1 \quad$ Nanotoxicology Research Lab, School of Engineering and Technology, Karunya Institute of Technology and Sciences, Coimbatore, Tamil Nadu 641114, India

2 Nanotechnology Innovation and Incubation Centre, PSG Institute of Advanced Studies, Coimbatore, Tamil Nadu 641004, India

3 Department of Biotechnology/Centre for Nanotechnology, Indian Institute of Technology-Roorkee, Roorkee, Uttarakhand 247667, India et al. 2011; Zanganeh et al. 2016; Kim et al. 2013; Baek et al. 2016). Superparamagnetism is a magnetic phenomenon pertaining to MNPs of appropriate size, having a single magnetic domain which behaves as a 'single super spin'. Such MNPs have a high magnetic susceptibility and hence an application of a magnetic field makes the MNPs respond strong and rapid to the field (Kodama 1999). On removal of the applied magnetic field, the MNPs do not possess residual magnetism at room temperature. Hence, they are not prone to agglomeration, which causes them escape phagocyte uptake and pose no unwarranted blockage of blood capillaries or thrombosis (Wahajuddin 2012). Iron oxide MNPs are the most commonly studied magnetic materials (Quinto et al. 2015) in the biomedical field. Apart from these, other ferrite MNPs have also been investigated (Mody et al. 2014; Choudhari et al. 2015; Hirosawa et al. 2017; Foroughi et al. 2016). Manganese ferrite NPs possess soft magnetic properties, viz., moderate saturation magnetization and low coercivity, besides good mechanical hardness and chemical stability (Carta et al. 2010). Though studied as a notable magnetic resonance imaging (MRI) contrast agent (Wang et al. 2014) and a theranostic (Wang et al. 2016), manganese ferrites do work as drug delivery vehicles (Zhao et al. 2015).

( 
The size, shape, and surface properties of MNPs need to be wisely tuned in order to achieve a suitable drug targeting efficiency. Particles of a size smaller than $10 \mathrm{~nm}$ or larger than $200 \mathrm{~nm}$ are removed from circulation by renal clearance and spleen or phagocyte uptake (Wahajuddin 2012). An MNP having a size in the range of $10-100 \mathrm{~nm}$ is suitable for longer circulation and escaping reticuloendothelial system of the human body. Particles having a hydrophobic surface suffer opsonisation (adsorption on protein surface) and macrophage uptake (Veiseh et al. 2010). On the contrary, surface-engineered nanoparticles having hydrophilic polymers as sheaths can escape reticuloendothelial cells or circulating macrophages (Harris and Chess 2003). As a result, they attain enhanced residence time in the blood. Surface charges on the polymers influence the ability to get directed to the target. For instance, cationic polyethyleneimine (PEI)-coated MNPs interact with deoxyribonucleic acid (DNA) (Steitz et al. 2007) and negatively-charged functional group-containing dextran-coated MNPs couple with peptide oligomers (Hildebrandt et al. 2007). Unlike these electrostatic interactions, affinity interactions through non-covalent binding are less affected by environmental conditions including changes in $\mathrm{pH}$ and ionic strength (Wahajuddin 2012).

One of the major limitations of drug delivery by loading drugs on surface-modified MNPs is the low entrapment efficiency of drug molecules. The other disadvantage is the failure in the release of drugs due to attachment of drugs through covalent linkages to the polymer shell (Wahajuddin 2012). The normal modes of drug release from polymers (Langer and Peppas 1981) are (1) surface erosion of the polymer and the diffusion of drug, (2) breaking of polymer bonds, and (3) physical diffusion of the encapsulated drug. At this juncture, the application of the cyclic oligosaccharides, cyclodextrins (CDs), find importance as a drug carrier. Cyclodextrins are doughnut-shaped molecules which can encapsulate a variety of drug molecules of appropriate size to fit into their cavity (Davis and Brewster 2004). CDs are non-toxic and water-soluble and possess a hydrophobic cavity and a hydrophilic exterior (Chandrasekaran et al. 2014; Yousuf et al. 2013). The drug encapsulated by CD is released in a slow and sustained manner (Davis and Brewster 2004).

With the objective to link the remarkable properties of poly(ethylene glycol) and $\beta$-cyclodextrin as drug carriers, there have been attempts to functionalize PEG with $\beta$-CD (Cesteros et al. 2006; Salmaso et al. 2007, 2004). The CD-PEG hybrid forms hydrogels and has been used in drug delivery (Cesteros et al. 2006). $\beta$-CD forms host-guest complex with the cancer drug, camptothecin (CPT) (Kang et al. 2002). On loading CPT in $\beta-C D$, the solubility and stability of the drug get enhanced. Moreover, the loading of CPT in $\beta$-CD enhances its antitumor potential (Cheng et al. 2004). The above-mentioned points intrigued framing of the present work being centered on (1) synthesis of manganese ferrite nanoparticles in a size range suitable for cancer drug targeting, (2) synthesis of CD-PEG conjugate, and (3) loading of CPT in the CD-PEG-coated MNPs (CD-PEG-MNPs@ CPT) and (4) the study of cytotoxicity of the MNP-CD-PEG polymer hybrid.

\section{Experimental}

\section{Materials}

The AnalaR reagents ( $p$-toluenesulfonic anhydride, $p$-toluenesulfonic chloride, ethylene diamine, and polyethylene glycol) used for the synthesis of the CD-derivative of polyethylene glycol were products of Aldrich, India. $\beta-C D$ was supplied by Hi-Media and polyethylene glycol by Fisher Scientific. All the solvents used for the synthesis (in AR grade) and for UV-Visible spectral measurements (in spectral grade) were purchased from Aldrich. The reagents used for the preparation of manganese ferrite MNPs, viz., manganese nitrate, ferric nitrate, ammonium hydroxide, and ethylene diamine were received from Merck, India. The purity of the chemicals was above $98 \%$. The dialysis membranes used for the drug release study were the products of Hi-Media, India (diameter $14.33 \mathrm{~mm}, \mathrm{LA} 387-1 \mathrm{MT}$ ). MTT (3-(4,5-dimethylthiazol-2-yl)-2,5-diphenyltetrazolium bromide) were procured from Amresco. The nuclear staining dye bis-Benzamide H 33342 trihydrochloride (Hoechst 33342) was purchased from Sigma-Aldrich. Cytoplasmic staining dye Rhodamine B was acquired from life technologies. The human embryonic kidney cells 293 (HEK 293) and human colon adenocarcinoma cells (HCT 15) were received from National Centre for Cell Science, Pune, India.

\section{Methods}

\section{Preparation of manganese ferrite MNPs}

Manganese ferrite was prepared using the procedure reported earlier (Mahmoodi 2013). Manganese nitrate (4.90 g, 1 eq.) and Ferric nitrate (13.4 g, 1.5 eq.) was dissolved in $50 \mathrm{~mL}$ distilled water and added to the aqueous mixed solution $[4.2 \mathrm{~g} \mathrm{NaOH}$ in $70 \mathrm{~mL}$ distilled water and $3 \mathrm{~mL}$ ethylenediamine (EDA)]. This solution was heated at $90{ }^{\circ} \mathrm{C}$ for $1 \mathrm{~h}$ to achieve complete chelation. The powder was calcined on alumina crucible at $500{ }^{\circ} \mathrm{C}$ for $1 \mathrm{~h}$, with a heating rate of $10^{\circ} \mathrm{C} / \mathrm{min}$.

\section{Synthesis of PEG-bis-(tosylate) (Ts-PEG-Ts)}

PEG-bis-(tosylate) was prepared using the procedure reported earlier (Hai et al. 2003). PEG (1.0 mL, 1 eq.) TEA 
( $2.9 \mathrm{~mL}, 2$ eq.) were mixed in $200 \mathrm{~mL}$ of dichloromethane and stirred at $40{ }^{\circ} \mathrm{C}$ for $30 \mathrm{~min}$. To this mixture, 2 eq. of tosyl chloride $(3.8 \mathrm{~g}$ ) was added to $100 \mathrm{~mL}$ of dichloromethane. The solution was constantly stirred using a magnetic stir bar at $40{ }^{\circ} \mathrm{C}$ for $5 \mathrm{~h}$. The product obtained was washed with ethyl acetate and dried.

\section{Synthesis of PEG-bis-(amine) $\left(\mathrm{H}_{2} \mathrm{~N}-\mathrm{PEG}-\mathrm{NH}_{2}\right)$}

PEG-bis-amine was prepared using a reported procedure (Hai et al. 2003). PEG-bis-sulfonate (1.0 g, $3 \mathrm{mmol}$ ) is dissolved with agitation in $29 \%$ aqueous ammonia to a concentration of between about $3 \mathrm{mmol}$ per litre and stirred for $1 \mathrm{~h}$. A dichloromethane is added to the reaction mixture and subsequently removed via phase separation. The solvent is dried and PEG-bis amine has precipitated the addition of diethyl ether.

\section{Synthesis of $\beta-C D-P E G$ conjugate}

Under nitrogen atmosphere, 6- $p$-toluenesulfonyl- $\beta$ cyclodextrin (Ts-CD, $0.5 \mathrm{~g}, 1$ eq.) and PEG-bis-(amine) ( $1.5 \mathrm{~g}, 1$ eq.) were mixed in $5 \mathrm{~mL}$ of DMSO and stirred at $60{ }^{\circ} \mathrm{C}$ for $12 \mathrm{~h}$. After cooling it to room temperature the mixture was poured into acetone. The precipitated product was filtered washed with acetone and dried.

\section{CD-PEG coating of MNPs}

The calcium ferrite MNPs were coated with the CD-PEG conjugate by adding $10 \mathrm{mg}$ of the former with $10 \mathrm{mg}$ of the latter in a slightly basic ( $\mathrm{pH}$ 8.0) water medium (by adding $0.1 \mathrm{M}$ sodium hydroxide in drops) and then sonicating to get CD-PEG-MNPs.

\section{Characterization}

A Shimadzu XRD 6000 instrument with a $\mathrm{Cu} \mathrm{K} \alpha$ radiation source $(\lambda=1.5418 \AA$ ) was used for measuring X-ray diffraction pattern with the generator operating at $40 \mathrm{kV}$. A JEOL JSM 6390 instrument was used to record scanning electron microscopic images. Transmission electron microscopic (TEM) images were recorded using a JEOL JSM 2100 instrument and the acceleration voltage applied was $200 \mathrm{kV}$. Dynamic light scattering (DLS) measurement was done using a Malvern Nano Zs90 Zetasizer. A qualitative estimation of the CD-PEG-MNPs was carried out using a Phi 5000 versa probe II base system X-ray photoelectron spectrometer. FT-IR spectra were recorded on a Shimadzu FT-IR spectrophotometer in the wavenumber range of $450-4000 \mathrm{~cm}^{-1}$. The sample was made as a pellet by crushing with KBr. A Netzsch STA 449 F3 Jupiter thermal analyzer was used to record the thermogravimetric profile of the PVA-CD-MNPs at a heating rate of $10{ }^{\circ} \mathrm{C} / \mathrm{min}$. Under $\mathrm{N}_{2}$ flow $(50 \mathrm{~mL} / \mathrm{min}$ ). A Pt-Rh wire served as the sensor of the device. Magnetization measurements were carried out using a Lake shore 7410 vibrating sample magnetometer (VSM). ${ }^{1} \mathrm{H}$ NMR spectrum was recorded on a Bruker Avance III spectrometer operating at an operating frequency of $400 \mathrm{MHz}$. A JOEL GC Mate spectrometer was employed to record the mass spectrum.

\section{Drug loading}

A pre-determined amount of the freeze-dried CPT-loaded MNPs was weighed on a microbalance and dispersed in $10 \mathrm{~mL}$ of PBS (pH 7.4). The concentration of CPT was calculated using UV-Visible spectral measurement. The observed wavelength was $368 \mathrm{~nm}$ with reference to a calibration curve. The percentage of loaded drug was calculated using the following equation (Zhu et al. 2009):

Percentage of loaded drug $=\frac{\text { Weight of Camp in MNPs }}{\text { Weight of MNPs }} \times 100 \%$.

\section{In vitro drug release}

CD-PEG-MNP@CPT was suspended in dialysis bags which were dipped in PBS buffer solution of $\mathrm{pH} 7.4$ and the other in that of $\mathrm{pH} 6.0$ and shaken using a mechanical shaker at $37{ }^{\circ} \mathrm{C}$. The solutions released outside the bags were withdrawn at regular time intervals and the concentration of the released drug was calculated using UV-Visible spectroscopy. The volume in the dialysis system was maintained constant by adding fresh PBS solutions. Three independent experiments were done and the average values were calculated.

\section{Cell culture}

The human embryonic kidney cells 293 (HEK 293) were grown in Dulbecco's modified eagle's medium (DMEM) and human colon adenocarcinoma cells (HCT 15) were maintained in Roswell Park Memorial Institute (RPMI) medium augmented with $10 \%$ fetal bovine serum (FBS, Gibco Life Technologies) and 1\% penicillin-streptomycin (FBS, Gibco Life Technologies) in both the cases and grown in $37{ }^{\circ} \mathrm{C}$ incubator with $5 \% \mathrm{CO}_{2}$ and $95 \%$ air with humidified condition provided.

\section{Cell viability assay}

The MTT assay was conducted for the evaluation of cytotoxicity while exposing the various drug-loaded nanoformulations (CD-PEG-MNP, CD-PEG-MNP@CPT). Briefly, cells were seeded uniformly in 96-well cell culture plates at

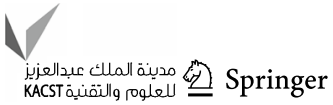


a seeding density of $7 \times 10^{4}$ cells/well for overnight incubation, and then the fresh medium with different nanoformulations (CD-PEG-MNP, CD-PEG-MNP@CPT), with respect to different concentrations, was added to the plate. These cells were incubated further for $24 \mathrm{~h}$. As the incubation time finished, the culture medium was terminated and cells underwent a phosphate-buffered saline (PBS) wash. To determine cytotoxicity, $100 \mu \mathrm{L}$ of fresh medium containing $10 \mu \mathrm{L}$ of MTT reagent $(5 \mathrm{mg} / \mathrm{mL}$ in PBS) was added to each well and incubated for $4 \mathrm{~h}$ for the formation of purple formazan crystals. Later, the medium was carefully withdrawn and dimethyl sulfoxide (DMSO-100 $\mu \mathrm{L} /$ well) was added to dissolve the formazan crystals. The absorbance at $570 \mathrm{~nm}$ of the purple formazan in each well was determined by using Cytation 3, Biotek multi-mode plate reader. The percentage cell viability of the untreated cells was presumed as $100 \%$. Relative cell viability [mean (\%) $\pm \mathrm{SEM}, n=3$ ] was assessed by the following equation:

$$
\begin{aligned}
\text { Cell viability }(\%)= & {[(\text { A570 }- \text { A690) for treated cells } /} \\
& (\text { A570 }- \text { A690) or control cells }] \times 100 .
\end{aligned}
$$

\section{Acridine orange-ethidium bromide (AO-EB) dual staining}

The AO-EB dual staining was performed to evaluate the apoptotic induction in cancer cells, which were preincubated with camptothecin-loaded nanoformulation (CD-PEG-MNPs@CPT). The growth medium was removed and PBS with $2 \mu \mathrm{L}$ of AO-EB mixture $(10 \mu \mathrm{g} / \mathrm{mL}$ of both were mixed) was used for staining and then incubated at $37^{\circ} \mathrm{C}$ for $5-10 \mathrm{~min}$. Then excess dyes were removed by PBS washing of the cells. Later, the stained cells were fixed with 3\% paraformaldehyde for $10 \mathrm{~min}$. The image of cellular morphology was then captured by EVOS FL Color, AMEFC 4300 cell imaging system (Life Technologies, USA) under the red fluorescent protein (RFP) filter for EB and green fluorescent protein (GFP) filter for $\mathrm{AO}$ as fluorescence images employing transmission mode for bright field images.

\section{Rhodamine B and Hoechst 33342 staining}

Combinatorial fluorescent stains (rhodamine B and Hoechst 33342) were used to visualize the intracellular morphological changes in the cytoplasm as well as the nucleus during the course of camptothecin-loaded nanoformulation treatment. Rhodamine B can be used to track the cytoplasmic vesicles and mitochondria effectively by staining the same and visualized under RFP filter. On the contrary, Hoechst 33342 nucleic acid staining dyes exclusively within the nucleus which can be tracked under DAPI filter. After the course of incubation, the cells treated with CD-PEG-MNPs@CPT were given a momentary PBS wash and then stained with $1 \mu \mathrm{L}$ of rhodamine B (working stock concentration: $1 \mathrm{mg} / \mathrm{mL}$ ) for $10 \mathrm{~min}$. Then, PBS wash was given to remove the excess rhodamine B. Finally, PBS accompanied with $2 \mu \mathrm{L}$ of Hoechst 33342 (working concentration: $10 \mathrm{mg} / \mathrm{mL}$ ) were added. Final washing was done with PBS followed by fixation with $3 \%$ paraformaldehyde, and the images were captured by EVOS cell-imaging system under DAPI, GFP and RFP filter. Overlay of images obtained in all three filters dictates the morphology of both the nucleus and the cytoplasm of HCT 15 cells.

\section{Results and discussion}

\section{Synthesis and characterization of CD-PEG}

Scheme 1 illustrates the steps in the synthesis of CD-PEG. The procedure is discussed in the "Experimental" section. The synthesized CD-PEG polymer was characterized using, IR, NMR, and mass spectral methods. The spectra are shown in the Electronic Supplementary Information ESI 1.

\section{Characterization of magnetic nanoparticles}

Figure 1a shows the XRD pattern of manganese ferrite nanoparticles (as-prepared and CD-PEG coated). The crystalline nature of the MNPs is revealed by the sharp peaks. The dif-

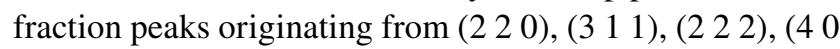

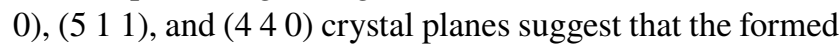
crystals are of spinel structure. The major peak harmonizes with the crystal reflection of MNPs in JCPDS CIF 1528316. The results from ICP-AES measure reveal that the exact composition of the ferrite MNPs is $\mathrm{Mn}_{0.98} \mathrm{Fe}_{2.03} \mathrm{O}_{4}$. The size of the grains can be calculated analyzing the (3 111$)$ diffraction peak, which is the most intense peak, using the Scherrer equation (Cullity and Stock 2014):

$t=0.9 \lambda / \beta \cos \theta$,

where $\beta$ refers to the full width of the diffraction line at half of the maximum intensity (radians), $\lambda$ is the wavelength of the X-ray employed, and $\theta$ is the Bragg angle. The calculated grain size is $11 \mathrm{~nm}$.

The EDX spectrum of the as-prepared MNPs is shown in Fig. 1b. There are peaks observed at various energy (keV) values which correspond to the elements $\mathrm{Mn}, \mathrm{Fe}$, and $\mathrm{O}$, indicating qualitatively the presence of these elements in the as-prepared ferrite sample and the absence of any other elements as impurities. However, we recorded XPS spectra for evaluating the CD-PEG-MNPs for a better preciseness of the elemental analysis, which will be discussed later in this section.

Figure 1c displays the TEM image of the CD-PEG-MNPs. The covered layer of the polymer is thin 


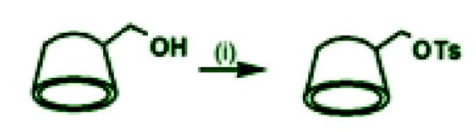

(1) $\mathrm{TsCl}, \mathrm{NaOH}, \mathrm{NH}_{4} \mathrm{Cl}$

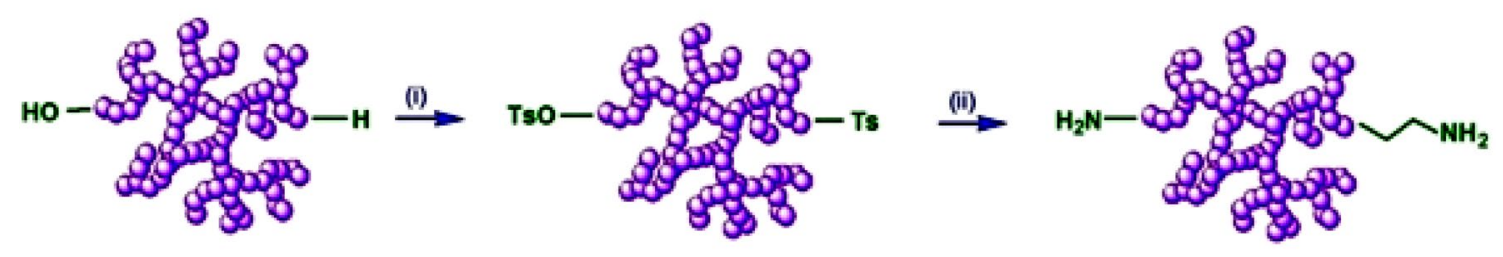

(i) $\mathrm{TsCl}$ (ii) $\mathrm{NH}_{4} \mathrm{OH}$

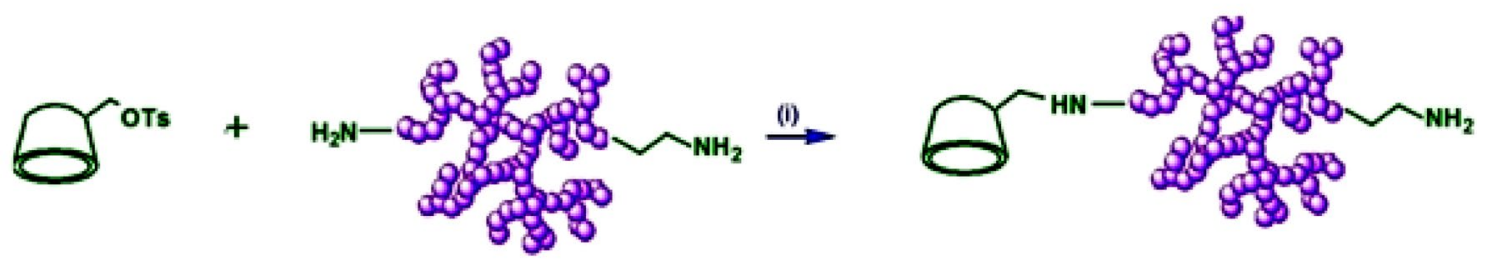

(i) $\Delta 60^{\circ} \mathrm{C}$

Scheme 1 Synthesis of CD-PEG conjugate

compared to the size of the MNPs. The average size of the MNPs is $93 \pm 5 \mathrm{~nm}$. This size is much larger than manganese ferrite NPs reported earlier for the purpose of MRI or other biomedical objective (Lemarchand et al. 2004; Leal et al. 2015; Beeran et al. 2015; Kang et al. 2004; Li et al. 2004; Giri et al. 2012; Mazarío et al. 2016). The hydrodynamic diameter of the CD-PEG-MNPs can be determined by dynamic light scattering (DLS) measurements. In the present case, the average hydrodynamic diameter observed is $95 \pm 5 \mathrm{~nm}$ as can be seen from the size distribution histogram shown in Fig. 1d.

After coating the MNPs with CD-PEG, the sample was used to record a thermogravimetric profile (Fig. 2). The TGA profile shows a two-step weight loss for the CD-PEG-MNPs.

An initial weight loss of nearly $10 \%$ is observed up to the temperature of $180{ }^{\circ} \mathrm{C}$, owing to the loss of moisture, the expulsion of the surface hydrated water from CD-PEG, and any possible volatile components present. The second weight loss occurs at $380{ }^{\circ} \mathrm{C}$, which is due to the decomposition of CD-PEG polymer. The next weight loss appears in between 600 and $800{ }^{\circ} \mathrm{C}$ due to the thermal degradation of the polymer. The TGA as carried out under an atmosphere of nitrogen in order to keep the oxidation reaction in the ferrite minimal. Hence, CD-PEG wraps around the MNPs and is considerably stable, without getting detached easily at lower temperatures.
To get additional evidence on the elemental composition and the ionic contribution to the ferrite MNPs, XPS spectra were recorded for the CD-PEG-MNPs. The XPS spectra show the peaks corresponding to the binding energies of electrons in Fe, and Mn, and O (See ESI 2). The spectra confirm the formation of crystalline manganese ferrite without impurity of other oxides. The explanations for each and every peak in the spectra are given in ESI 2.

\section{Magnetic properties}

The room temperature magnetic behaviour of the as-prepared and the CD-PEG-MNPs was studied by recording the magnetization vs applied field plot (Fig. 3). In both cases, the hysteresis loop is absent, i.e., the coercivity is almost zero. The saturation magnetization of the as-prepared MNPs is $38 \mathrm{emu} \mathrm{g}^{-1}$, typical of the soft magnetic manganese ferrite MNPs. Coating of CD-PEG results in the saturation magnetization decreasing by $5 \mathrm{emu}^{-1}$, which is due to the non-magnetic characterization of the coating polymer. The small decrease is due to just a thin shell of polymer covering up the MNPs core. The magnetization value of the MNPs is larger than that reported for $\mathrm{MnFe}_{2} \mathrm{O}_{4}$ by some authors (Pal et al. 2014; Naseri et al. 2011; Fahmi et al. 2015) but close to some other reports for the same nanomaterial (Vestal and Zhang 2003; Aslibeiki et al. 2016). The difference arises due to the size variations between the reported magnetic

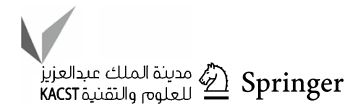



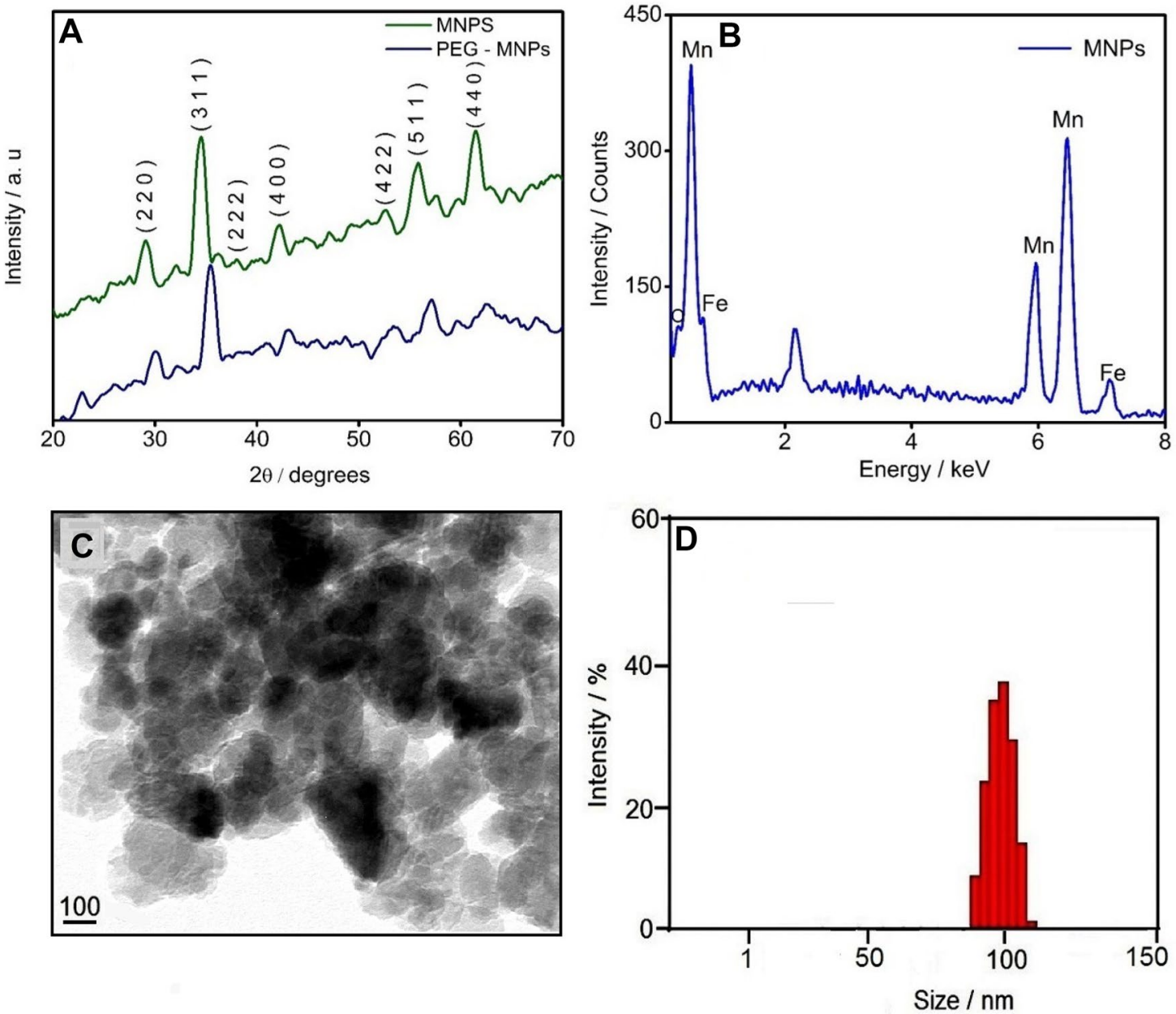

Fig. 1 a X-ray diffraction patterns of a as-prepared MNPs (b) CDPEG-MNPs. b Energy-dispersive X-ray spectrum of as-prepared MNPs. c Transmission electron microscopic image of CD-PEG-

MNPs. d Particle size distribution of CD-PEG-MNPs observed from dynamic light scattering
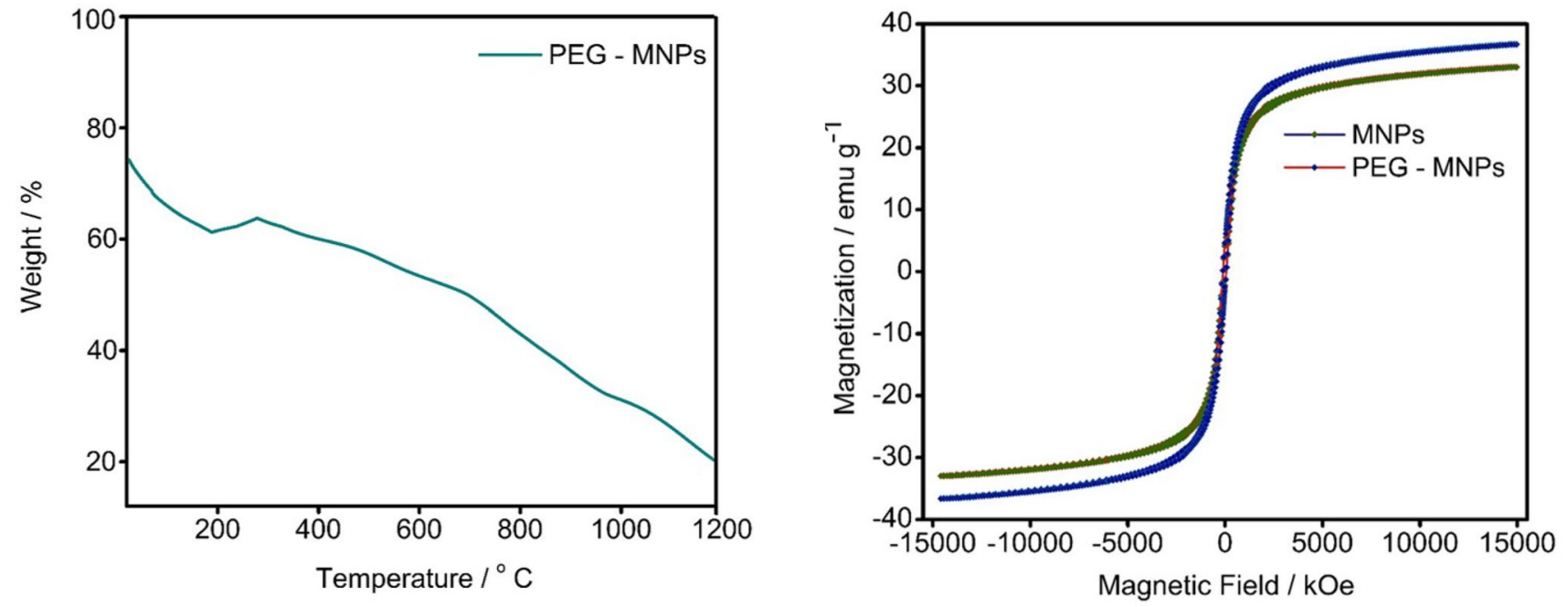

Fig. 2 Thermogravimetric curves of the CD-PEG-MNPs

Fig. 3 Magnetization curves of MNPs 
nanoparticles. The absence of hysteresis loop suggests a room temperature superparamagnetic behaviour and the size of the approximately $99 \mathrm{~nm}$ MNPs are within the superparamagnetic limit.

\section{Drug loading and release}

We calculated the percentage of drug loading on the CD-PEG-MNPs@CPT using Eq. (1). A pre-calculated amount of CD-PEG-MNPs@CPT was dispersed in $10 \mathrm{~mL}$ of water. The concentration of CPT was calculated by UV-Vis absorption (385 nm). Comparatively, the percentage of loading and efficiency of adsorption on unmodified PEGcoated MNPs (PEG-MNPs) were determined to employ the above equation, in order to comprehend the role of $\beta-C D$ conjugation in drug encapsulation. The percentages of the loaded drug on CD-PEG-MNPs@CPT and PEG-MNPs are 86 and $60 \%$, respectively.

Obliviously, the loading drug content in $\beta$-CD modified PEG is more compared to the unmodified PEG. The CPT molecule enters the space between the folded strands of PEG and resides inside the polymer shell. Moreover, it is plausible that the hydroxyl group forms hydrogen bonds with the -OH group of PEG. The weak association allows the release of drug from the polymer shell of MNPs. An enhanced loading content in the CD-PEG-MNPs@CPT suggests that the $\beta$-CD encapsulates the CPT molecule through host-guest association. Due to the larger room for accommodating the drug molecule, the enhanced loading occurs. This inference is future supported by the fact that native $\beta$-CD form host-guest complex with CPT (Kang et al. 2002). It is noteworthy that the adsorption efficiency of both the PEG-MNPs and CD-PEG-MNPs are generally large and suitable for transport of the drug cargo.
The in vitro release of CPT was studied following the procedure reported by Zhu et al. (2009). Suspensions of CD-PEG-MNPs@CPT were taken in dialysis membranes having a cut-off for molecular weight range of 12,000-14,000 Da. The membranes placed in PBS solution in two different pHs, viz., 6.0 and 7.4 were put in a shaker bath. The release of the drug was studied at two different temperatures, viz., 35 and $40{ }^{\circ} \mathrm{C}$. UV-Vis absorption measurements were made at regular and planned intervals of time. The in vitro release profiles of CD-PEG-MNPs@ CPT are shown in Fig. 4. The release profile shows that nearly $30 \%$ of the drug is released at pH 7.4 in $200 \mathrm{~min}$ (Fig. 4a). Further, the release rate is slow and sustained and comes up to a plateau at $800 \mathrm{~min}$. Evan at this time, the released amount of drug is $54.5 \%$ and the release further continues steadily. A decrease in $\mathrm{pH}$ accelerates the drug release from $54.5 \%$ at $\mathrm{pH} 7.4$ to $69.4 \%$ at $\mathrm{pH} 6.0$. The two mentioned pH's are chosen for the experiments since 7.4 is the normal physiological and the $\mathrm{pH}$ is decreased in the tumour microenvironment due to acidic metabolites caused by anaerobic glycolysis in hypoxia (Feng et al. 2018). The larger size of nanoparticles $(>100 \mathrm{~nm}$ ) results in a slower release of loaded drugs (Zhu et al. 2009). In the present case, even though the size is smaller, the drug release is slow and sustained, which is attributed to the greater drug-carrying ability of the polymer shell.

The increase of temperature (to $40{ }^{\circ} \mathrm{C}$ ) results in a quicker release of CPT (Fig. 4b), i.e., $40 \%$ of the drug in nearly $200 \mathrm{~min}$. In this temperature, for the sample at $\mathrm{pH}$ 6.0 , the release rate further gets enhanced. An elevation in temperature, hence leads to a better release of the encapsulated drug, leading to the hyperthermia based tuning of drug release from the transporting vehicle i.e., MNPs.
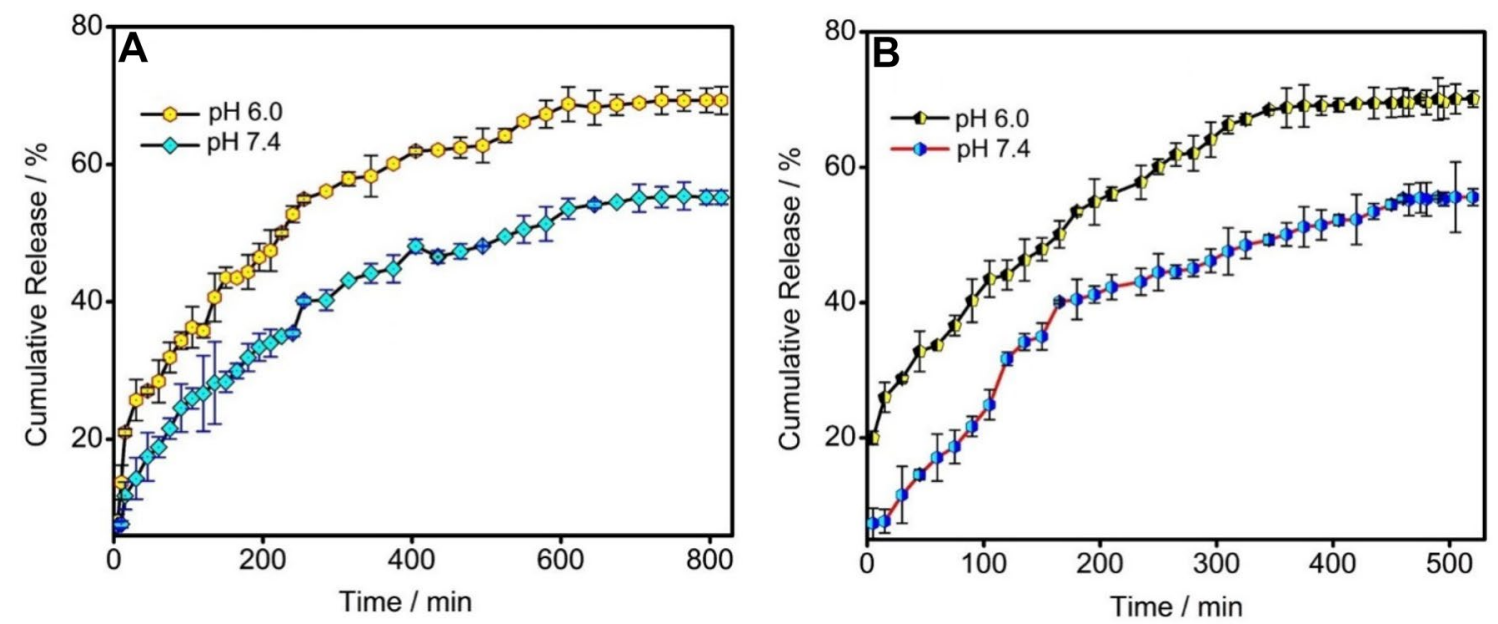

Fig. 4 a Release profile of camptothecin loaded on CD-PEG-MNPs under the pH conditions of 7.4 and 6.0 at room temperature. b Release profile at $40{ }^{\circ} \mathrm{C}$

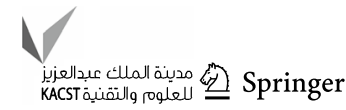




\section{Cell viability assay}

The cytotoxic effects of CD-PEG-MNPs and CD-PEG-MNPs@CPT were screened on HCT-15 and HEK293 quantitatively by MTT assay as shown in the Fig. 5a, b, respectively.

In the comparative analysis, the cytotoxicity was found to be increased when the drug was loaded in the CD-PEG-MNPs in both normal and cancer cell lines. These data reveal that the anti-neoplastic potential of camptothecin can be successfully utilized in MNPs for drug delivery applications.

This assay later provided the knowledge of $\mathrm{IC}_{50}$ values for different nanoformulations. From the results, CD-PEG-MNPs are found to have an $\mathrm{IC}_{50}$ value of 32.7 and $7.21 \mu \mathrm{g} / \mathrm{mL}$ for HCT-15 and HEK293 cells individually. The cytotoxicity found in HEK 293 cell is comparatively higher than in HCT-15 cells in the material toxicity concern.
The multi-drug resistant nature of colon cancer cells (HCT15) might be the reason behind the discrimination in toxicity. The involvement of multiple, molecular mechanisms to escape the drug burden (Uchiyama-Kokubu and Watanabe 2001) was found in the cancer regime, whereas the non-cancerous cell types do not possess such a mechanism and are more sensitive to the stress caused by therapeutic dosage. To substantiate the carrier toxicity and establish a potential drug delivery system, we compared the bare CD-PEG-MNPs and CD-PEG-MNPs@CPT on HCT-15 cell line. As expected, the bare CD-PEG-MNPs displayed a lower toxicity whereas CD-PEG-MNPs@CPT resulted with an $\mathrm{IC}_{50}$ value of $19.3 \mu \mathrm{g} / \mathrm{mL}$. The manganese toxicity (Hernández et al. 2011) may be influenced by the cytotoxicity of bare CD-PEG-MNPs irrespective of cell type discrimination as in normal and cancer cell lines.
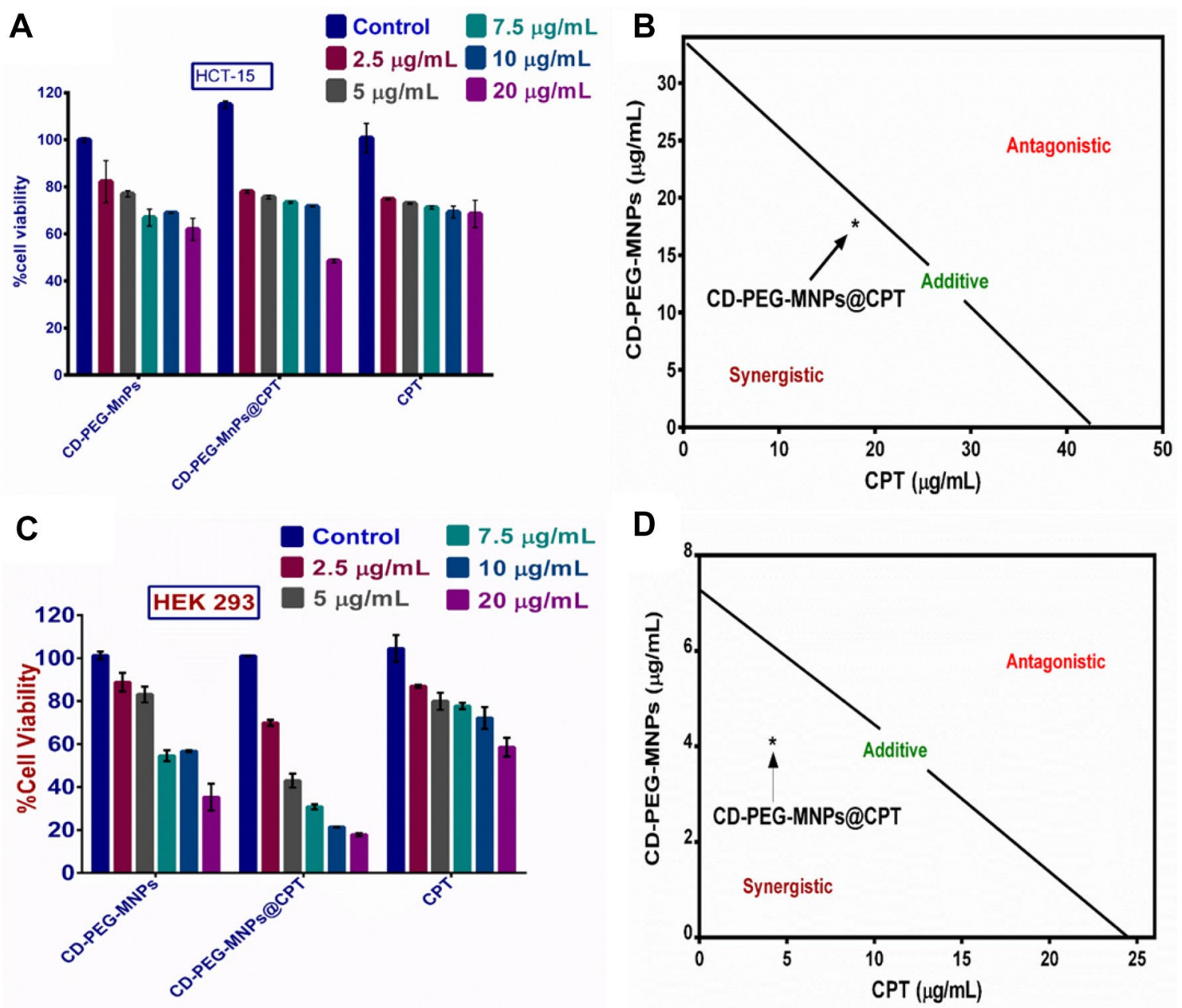

Fig. 5 The cell viability of a HCT-15 cells and c HEK 293 cells obtained by MTT assay upon treatment with CD-PEG-MNPs, CDPEG-MNPs@CPT and CPT on a course of $24 \mathrm{~h}$. The values are represented as mean \pm S.E.M. $(n=3)$. The isobolograms were drawn for interpreting the synergy analysis between CD-PEG-MNPs and CPT as constituents of CD-PEG-MNPs@CPT in b HCT-15 cells and d HEK 293 cells, respectively 


\section{Isobologram analysis}

The cytotoxicity of drug-loaded MNPs was further evaluated in order to know cytotoxic-determining factors in the nanoformulation. The synergistic effect of drug alone (camptothecin) and MNPs was compared with respect to the drug-loaded metal nanocomposite (CD-PEG-MNPs@ CPT). It was closely evidenced with a synergistic cytotoxic effect by the CD-PEG-MNPs@CPT. The data point of CD-PEG-MNPs@CPT in isobologram analysis was found to be way below additive lines in both the cell lines. The combined effect of MNPs and drug attributed to the strong synergistic in toxicity concerns (Fig. 5b, d). The augmented potential drug delivery strategy of the metal nanocomposite was explored in the assay and leads the study into appreciable efficacy.

\section{Acridine orange-ethidium bromide (AO-EB) staining}

To critically evaluate the mechanism of cell death by CD-PEG-MNPs@CPT, a combinatorial staining procedure was followed in HCT-15 cell line and observed under the fluorescence microscope. Viable healthy cells can eliminate EB stain. So nucleus of such cells appears in green because of AO fluorescence alone (Fig. 6a). The cells underwent treatment with the $\mathrm{IC}_{50}$ concentration of CD-PEG-MNPs@ CPT showed significant morphometric changes of the cell and owing apoptotic cells (Matai et al. 2015) (Fig. 6b).

\section{Rhodamine B and Hoechst 33342 staining}

Rhodamine B and Hoechst 33342 can be effectively used to stain the cytoplasm as well as the nucleus to detect the morphological changes after the incubation of CD-PEG-MNPs@CPT nanoformulations. Consecutive cascade events of apoptosis were assessed in HCT-15 incubated with CD-PEG-MNPs@CPT nanoformulations. The drastic reduction in the rhodamine B fluorescence in the cytoplasm's of the CD-PEG-MNPs@CPT (Fig. 7b) treated cells clearly suggested cytoplasmic constrictions, further in the same order while compared with untreated controls (Fig. 7a). Moreover, non-uniform spotted blue stains of Hoechst 33342 in nucleus provided the clue for nuclear fragmentation in treated cells (Fig. 7b), which stains the nucleus uniformly in untreated control (Fig. 7a) (Kumar and Gopinath 2015). The methods involved in the work are summarized in Scheme 2.

\section{Conclusions}

In this work, a new combination $\mathrm{Mn}_{0.98} \mathrm{Fe}_{2.02} \mathrm{O}_{4}-\beta$-cyclodextrin-PEG conjugate hybrid is developed. The size of the MNPs is $\sim 90 \mathrm{~nm}$, which is suitable for magnetic drug targeting as the nanomaterial, with this size, can escape renal clearance and uptake by the reticuloendothelial system. The CD-PEG-coated MNPs are superparamagnetic which is suitable for rapid magnetization on the application of external magnetic field and demagnetization when it is lifted. The percentage of loading of camptothecin gets enhanced from $60 \%$ in MNPs coated with unmodified PEG to $86 \%$ in CD-PEG-coated ones. The release of the drug from CD-PEG-MNPs is slow and sustained up to $800 \mathrm{~min}$. A decrease of $\mathrm{pH}$ from 7.4 to 6.0 and an increase of temperature both lead to the faster release of the drug, which enables stimuli-responsive release from the nanocarrier. The cytotoxicity of the bare CD-PEG-MNPs in HEK 293 and HCT 15 cell lines is lesser than that of the camptothecin-loaded ones. With the PEG aiding a possible escape from macrophage uptake, $\beta$-CD facilitating enhanced drug loading and sustained release, and the ferrite MNPs enabling magnetic field-directed targeting, the CD-PEG
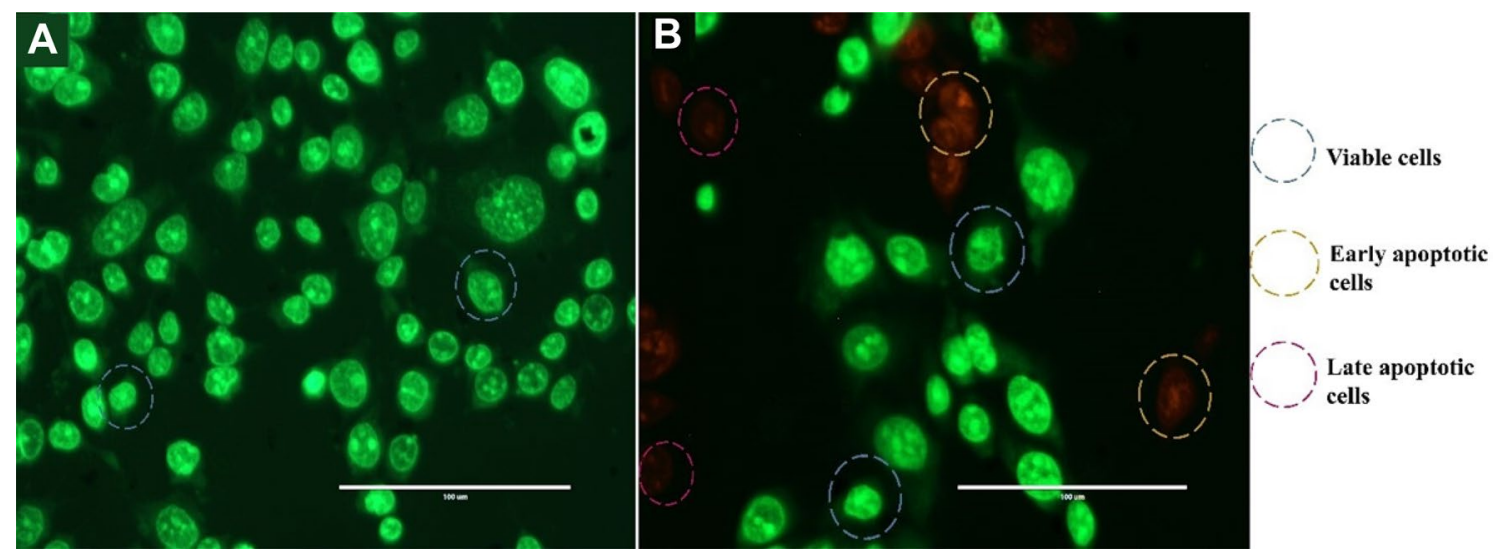

Fig. 6 AO-EB stained HCT-15 cells were showing progressive stages of apoptosis after $24 \mathrm{~h}$ incubation. a Untreated control, b IC 50 concentration of the CD-PEG-MNPs@CPT in respective green and red filters. Scale bar $100 \mu \mathrm{m}$ 

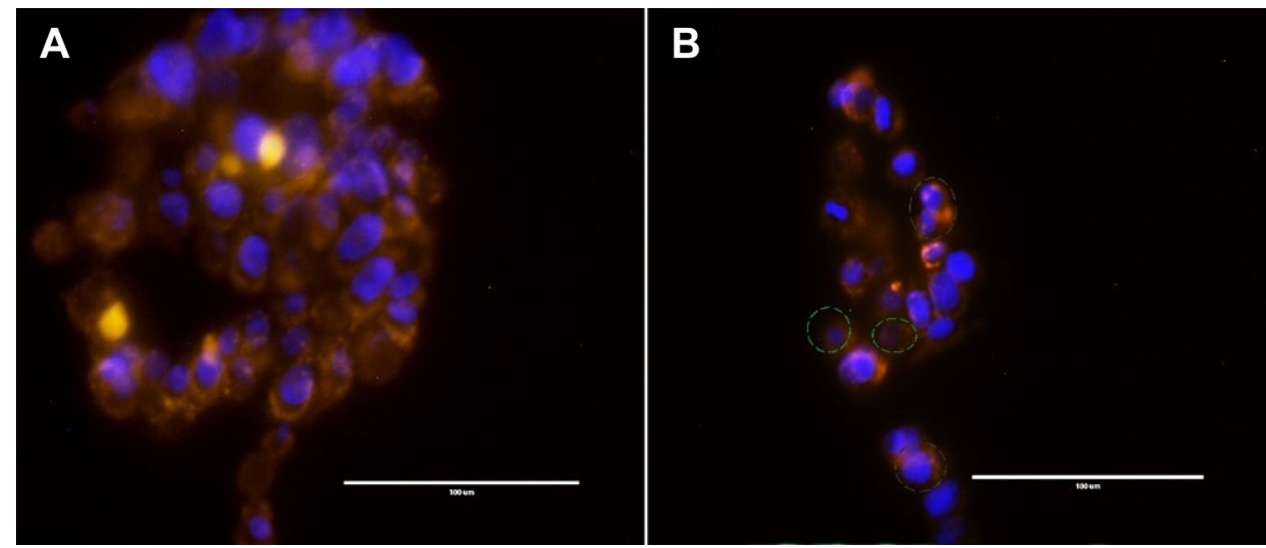

Cytoplasmic

constriction

Nuclear

fragmentation

Fig. 7 Hoechst 33342 and rhodamine B stained HCT-15 cells were showing morphology of apoptosis after $24 \mathrm{~h}$ incubation. a Untreated control, b $\mathrm{IC}_{50}$ concentration of CD-PEG-MNPs@CPT in respective blue and red filters. Scale bar $100 \mu \mathrm{m}$

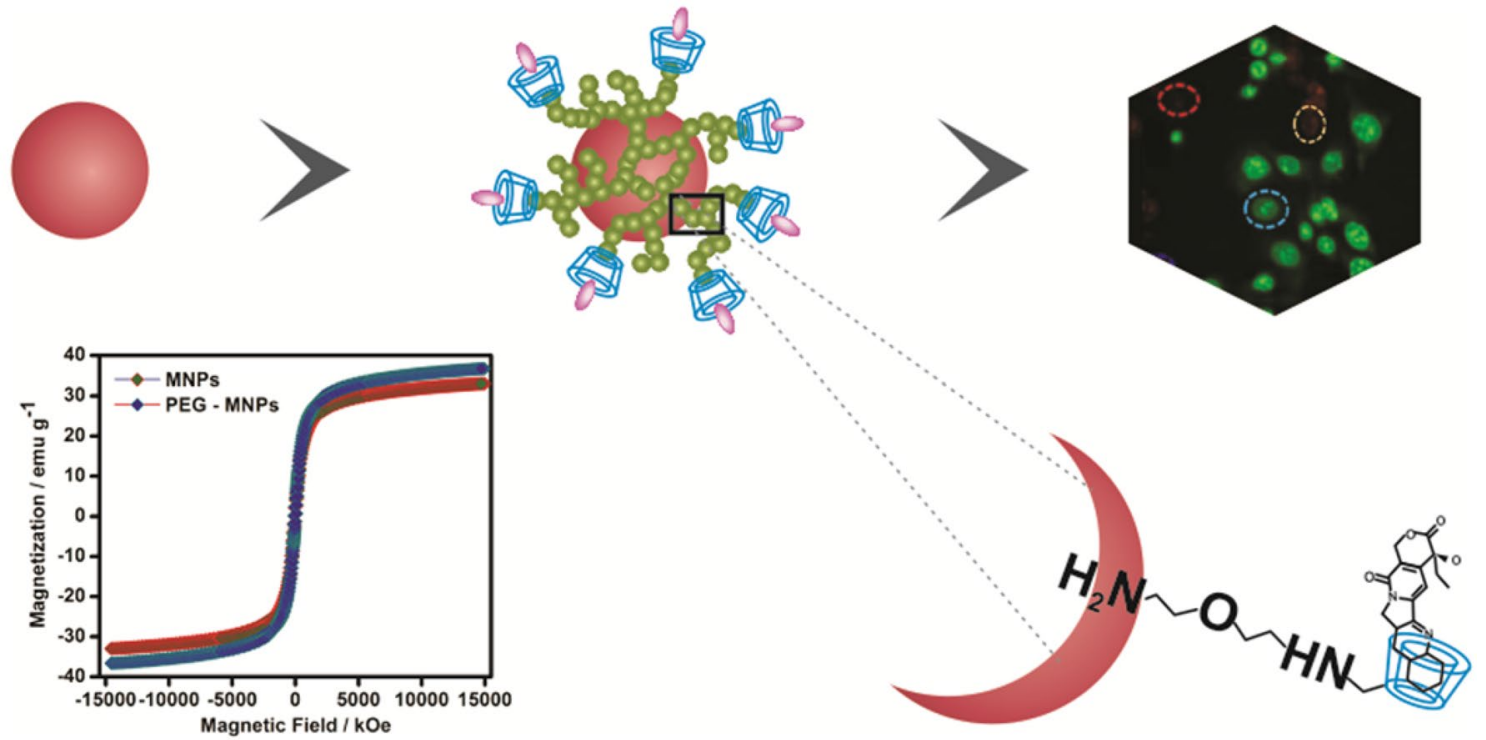

Scheme 2 Schematic representation of the CD-PEG-MNPs@CPT. Red sphere denotes the manganese ferrite NPs. $\beta$-CD is shown in bucket shape

wrapped MNPs will be a promising drug carrier for sharpshooting cancer cells.

Acknowledgements IVMVE thanks the management of Karunya University for the assistance in setting up a new Nanotoxicology lab.

\section{References}

Aslibeiki B, Kameli P, Ehsani MH, Salamati H, Muscas G, Agostinelli E, Foglietti V, Casciardi S, Peddis D (2016) Solvothermal synthesis of $\mathrm{MnFe}_{2} \mathrm{O}_{4}$ nanoparticles: the role of polymer coating on morphology and magnetic properties. J Magn Magn Mater 399:236-244
Baek SM, Singh RK, Kim TH, Seo JW, Shin US, Chrzanowski W, Kim HW (2016) Triple hit with drug carriers: pH- and temperature-responsive theranostics for multimodal chemo- and photothermal therapy and diagnostic applications. ACS Appl Mater Interfaces 8:8967-8979

Beeran AE, Fernandez FB, Nazeer SS, Jayasree RS, John A, Anil S, Vellappally S, Al Kheraif AA, Varma PR (2015) Multifunctional nano manganese ferrite ferrofluid for efficient theranostic application. Colloids Surf B 136:1089-1097

Carta D, Casula MF, Floris P, Falqui A, Mountjoy G, Boni A, Sangregorio C, Corrias A (2010) Synthesis and microstructure of manganese ferrite colloidal nanocrystals. Phys Chem Chem Phys 12:5074-5083

Cesteros LC, Ramírez CA, Pecina A, Katirie I (2006) Poly(ethylene glycol- $\beta$-cyclodextrin) gels: synthesis and properties. J Appl Polym Sci 102:1162-1166 
Chandrasekaran S, Sameena Y, Enoch IV (2014) Tuning the binding of coumarin 6 with DNA by molecular encapsulators: effect of $\beta$-cyclodextrin and C-hexylpyrogallol[4]arene. J Mol Recognit 27:640-652

Cheng J, Khim KT, Davis ME (2004) Antitumor activity of $\beta$-cyclodextrin polymer-camptothecin conjugates. Mol Pharm $1: 183-193$

Choudhari PR, Gaikwad VM, Acharya SA (2015) Role of mode of heating on the synthesis of nanocrystalline zinc ferrite. Appl Nanosci 5:711-717

Cullity BD, Stock SR (2014) Elements of X-ray diffraction. AddisonWesley, New York

Davis ME, Brewster ME (2004) Cyclodextrin-based pharmaceutics: past, present and future. Nat Rev Drug Discov 3:1023-1035

Fahmi MZ, Chen JK, Huang CC, Ling YC, Chang JY (2015) Phenylboronic acid-modified magnetic nanoparticles as a platform for carbon dot conjugation and doxorubicin delivery. J Mater Chem B 3:5532-5543

Feng L, Dong Z, Tao D, Zhang Y, Liu Z (2018) The acidic tumor microenvironment: a target for smart cancer nano-theranostics. Natl Sci Rev 5:269-286

Foroughi F, Hassanzadeh-Tabrizi SA, Bigham A (2016) In situ microemulsion synthesis of hydroxyapatite- $\mathrm{MgFe}_{2} \mathrm{O}_{4}$ nanocomposite as a magnetic drug delivery system. Mater Sci Eng C 68:774-779

Giri A, Goswami N, Bootharaju MS, Xavier PL, John R, Thanh NTK, Pradeep T, Ghosh B, Raychaudhuri AK, Pal SK (2012) Emergence of multicolor photoluminescence in $\mathrm{La}_{0.67} \mathrm{Sr}_{0.33} \mathrm{MnO}_{3}$ nanoparticles. J Phys Chem C 116:25623-25629. https://doi. org/10.1021/jp308606a

Hai T, Markoski L, Pereira D, Nordhaus M (2003) US 2003/0149307 A1

Harris JM, Chess RB (2003) Effect of pegylation on pharmaceuticals. Nat Rev Drug Discov 2:214-221

Hernández RB, Farina M, Espósito BP, Souza-Pinto NC, Barbosa F Jr, Sunol C (2011) Mechanisms of manganese-induced neurotoxicity in primary neuronal cultures: the role of manganese speciation and cell type. Toxicol Sci 124:414-423

Hildebrandt N, Hermsdorf D, Signorell R, Schmidtz SA, Diederichsena U (2007) Superparamagnetic iron oxide nanoparticles functionalized with peptides by electrostatic interactions. ARKIVOC 79:90

Hirosawa F, Iwasaki T, Watano S (2017) Synthesis and magnetic induction heating properties of $\mathrm{Gd}$-substituted $\mathrm{Mg}-\mathrm{Zn}$ ferrite nanoparticles. 7:209-214

Kang J, Kumar V, Yang D, Chowdhury PR, Hohl RJ (2002) Cyclodextrin complexation: influence on the solubility, stability, and cytotoxicity of camptothecin, an antineoplastic agent. Eur J Pharm Sci 15:163-170

Kang E, Park J, Hwang Y, Kang M, Park JG, Hyeon T (2004) Direct synthesis of highly crystalline and monodisperse manganese ferrite nanocrystals. J Phys Chem B 108:13932-13935

Kim DH, Vitol EA, Liu J, Balasubramanian S, Gosztola D, Cohen EE, Novosad V, Rozhkova EA (2013) Stimuli-responsive magnetic nanomicelles as multifunctional heat and cargo delivery vehicles. Langmuir 29:7425-7432

Kodama RH (1999) Magnetic nanoparticles. J Magn Magn Mater 200:359-372

Kumar SU, Gopinath P (2015) Controlled delivery of bPEI-niclosamide complexes by PEO nanofibers and evaluation of its antineoplastic potentials. Colloids Surf B 131:170-181

Langer RS, Peppas NA (1981) Present and future applications of biomaterials in controlled drug delivery systems. Biomaterials 2:201-214

Leal MP, Rivera-Fernandex S, Franco JM, Pozo D, dela Fuente JM, Garcia-Martinn ML (2015) Long-circulating PEGylated manganese ferrite nanoparticles for MRI-based molecular imaging. Nanoscale 7:2050-2059
Lee JH, Jang J, Choi J, Moor SH, Noh S, Kim J, Kim J, Kim I, Park KI, Cheon J (2011) Exchange-coupled magnetic nanoparticles for efficient heat induction. Nat Nanotechnol 6:418-422

Lemarchand C, Gref R, Couvreur P (2004) Polysaccharide-decorated nanoparticles. Eur J Pharm Biopharm 58:327-341

Li F, Zhang L, Evans DG, Duan X (2004) Structure and surface chemistry of manganese-doped copper-based mixed metal oxides derived from layered double hydroxides. Colloids Surf A 244:169-177

Mahmoodi NM (2013) Manganese ferrite nanoparticle: synthesis, characterization and photocatalytic dye degradation ability. Desalination Water Treat 53:84-90

Matai I, Sachdev A, Gopinath P (2015) Multicomponent 5-fluorouracil loaded PAMAM stabilized-silver nanocomposites synergistically induce apoptosis in human cancer cells. Biomater Sci 3: 457-468

Mazarío E, Mayoral A, Salas E, Menéndez N, Herrasti P, SánchezMarcos J (2016) Synthesis and characterization of manganese ferrite nanoparticles obtained by electrochemical/chemical method. Mater Des 111:646-650

Mody VV, Cox A, Shah S, Singh A, Bevins W, Parihar H (2014) Magnetic nanoparticle drug delivery systems for targeting tumor. Appl Nanosci 4:385-392

Naseri MG, Saion EB, Ahangar HA, Hashim M, Shaari AH (2011) Synthesis and characterization of manganese ferrite nanoparticles by thermal treatment method. J Magn Magn Mater 323:1745-1749

Pal M, Rakshit R, Mandal K (2014) Surface Modification of $\mathrm{MnFe}_{2} \mathrm{O}_{4}$ nanoparticles to impart intrinsic multiple fluorescence and novel photocatalytic properties. ACS Appl Mater Interfaces 6:4903-4910

Quinto CA, Mohindra P, Tong S, Bao G (2015) Multifunctional superparamagnetic iron oxide nanoparticles for combined chemotherapy and hyperthermia cancer treatment. Nanoscale 7:12728-12736

Salmaso S, Semenzato A, Caliceti P, Hoebeke J, Sonvico F, Dubernet C, Couvreur P (2004) Specific antitumor targetable beta-cyclodextrin-poly(ethylene glycol)-folic acid drug delivery bioconjugate. Bioconjugate Chem 15:997-1004

Salmaso S, Semenzato A, Caliceti P, Matricardi P, Rossi F, Caliceti P (2007) Cyclodextrin/PEG based hydrogels for multi-drug delivery. Int J Pharm 345:42-50

Steitz B, Hofmann H, Kamau SW, Hassa PO, Hottiger MO, von Rechenberg B, Hofmann-Amtenbrink M, Petri-Fink A (2007) Magnetic and optical properties of spinel $\mathrm{Fe}_{x} \mathrm{Co}_{3-x} \mathrm{O}_{4}$ thin films. J Magn Magn Mater 311:300-305

Uchiyama-Kokubu N, Watanabe T (2001) Establishment and characterization of adriamycin-resistant human colorectal adenocarcinoma HCT-15 cell lines with multidrug resistance. Anti-cancer Drugs 12:769-779

Veiseh O, Gunn JW, Zhang M (2010) Design and fabrication of magnetic nanoparticles for targeted drug delivery and imaging. Adv Drug Deliv Rev 62:284-304

Vestal CR, Zhang ZJ (2003) Synthesis and magnetic characterization of $\mathrm{Mn}$ and Co spinel ferrite-silica nanoparticles with tunable magnetic core. Nano Lett 3:1739-1743

Wahajuddin SA (2012) Superparamagnetic iron oxide nanoparticles: magnetic nanoplatforms as drug carriers. Int J Nanomed 7:3445-3471

Wang Z, Liu J, Li T, Liu J, Wang B (2014) Controlled synthesis of $\mathrm{MnFe}_{2} \mathrm{O}_{4}$ nanoparticles and $\mathrm{Gd}$ complex-based nanocomposites as tunable and enhanced $T_{1} / T_{2}$-weighted MRI contrast agents. J Mater Chem B 2:4748-4753

Wang L, Yan Y, Wang M, Yang H, Zhou Z, Peng C, Yang S (2016) An integrated nanoplatform for theranostics via multifunctional coreshell ferrite nanocubes. J Mater Chem B 4:1908-1914

Yousuf S, Sudha N, Murugesan G, Enoch IVMV. (2013) Isolation of Prunin from the fruit shell of Bixa orellana and the effect of

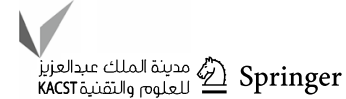


$\beta$-cyclodextrin on its binding with calf thymus DNA. Carbohyd Res 365:46-51

Zanganeh S, Hutter G, Spitler R, Lenkov O, Mahmoudi M, Shaw A, Pajarien JS, Nejadnik H, Goodman S, Moseley M, Coussens LM, Daldrup-Link HE (2016) Iron oxide nanoparticles inhibit tumour growth by inducing pro-inflammatory macrophage polarization in tumour tissues. Nat Nanotechnol 11:986-994

Zhao H, Chen Z, Tao L, Zhu X, Lan M, Li Z (2015) In vitro toxicity evaluation of ultra-small $\mathrm{MFe}_{2} \mathrm{O}_{4}(\mathrm{M}=\mathrm{Fe}, \mathrm{Mn}, \mathrm{Co})$ nanoparticles using A549 cells. RSC Adv 5:68454-68460
Zhu A, Yuan L, Jin W, Dai S, Wang Q, Xue Z, Qin A (2009) Polysaccharide surface modified $\mathrm{Fe}_{3} \mathrm{O}_{4}$ nanoparticles for camptothecin loading and release. Acta Biomater 5:1489-1498

Publisher's Note Springer Nature remains neutral with regard to jurisdictional claims in published maps and institutional affiliations. 